\title{
Line asymmetry in the Seyfert Galaxy NGC 3783
}

\author{
J M Ramírez and Manuel Bautista \\ Physics Center, Instituto Venezolano de Investigaciones Cientificas (IVIC), PO Box 21827, \\ Caracas 1020A, Venezuela \\ and \\ Timothy Kallman \\ NASA Goddard Space Flight Center, code 662, Greenbelt, MD 20771, USA
}

\begin{abstract}
We have reanalyzed the $900 \mathrm{ks}$ Chandra X-ray spectrum of NGC 3783, finding evidence on the asymmetry of the spectral absorption lines. The lines are fitted with a parametric expression that results from an analytical treatment of radiatively driven winds. The line asymmetry distribution derived from the spectrum is consistent with a non-spherical outflow with a finite optical depth. Within this scenario, our model explains the observed correlations between the line velocity shifts and the ionization parameter and between the line velocity shift and the line asymmetry. The present results may provide a framework for detailed testing of models for the dynamic and physical properties of warm absorber in Seyfert galaxies.
\end{abstract}

Subject headings: AGNs-absorption lines-ionized gas

\section{Introduction}

Since the launch of Chandra and XMM-Newton our view of active galaxies (AGNs) in X-rays has changed dramatically. It is now common to see rich and complex spectra in emission and absorption in the $0.5-10 \mathrm{keV}$ band, indicative of absorption by partially ionized gas. The warm absorber hallmark, which appears in about $50 \%$ of AGN spectra, has been recognized since the Einstein observation of QSQ MR 2251+178 (Halpern 1984) and the subsequent detection of the $\mathrm{O}$ VIII $(871 \mathrm{eV})$ and $\mathrm{O}$ VII $(739 \mathrm{eV})$ absorption edges by ROSAT and $A S C A$ (Reynolds 1997; George et al. 1998; Nandra \& Pounds 1992; Fabian et al. 1994). Then, the complexity of AGN spectra become more evident thanks to the improvement in 
resolution and sensitivity of the instruments on board the new generation of X-ray telescopes; for example the HETGS (High-Energy Transmission Grating Spectrometer; Canizares et al. 2000) with resolution of $0.012-0.023 \AA$ on board Chandra. This allows us to study in detail the physical structure and the dynamical conditions of the nuclear environment in Quasars and Seyfert galaxies. The best example of this so far is NGC 3783, which shows edges and absorption lines coming from $\mathrm{H}$ - and He-like ions of $\mathrm{Si}, \mathrm{S}, \mathrm{Mg}, \mathrm{Ne}, \mathrm{O}, \mathrm{N}$ as well as $\mathrm{L}$ - and M-shell Fe transitions (Kaspi et al. 2002).

One of the primary goals of all AGN studies is to gain an understanding of the gas flows in the vicinity of the central black hole. The X-ray absorption lines seen in NGC 3783 are blueshifted with respect to the rest-frame of the host galaxy by moderate $\sim 1000 \mathrm{~km} / \mathrm{s}$, velocity (Kaspi et al. 2001, 2002). UV observations with the Space Telescope Imaging Spectrograph (STIS) on the Hubble Space Telescope and the Far Ultraviolet Spectroscopic Explorer (FUSE), have also revealed blueshifted absorption features coming from O VI, N V, C IV, $\mathrm{N}$ III and C III lines (Gabel et al. 2003a,b), providing a conclusive signature of outflow. This outflow character must be incorporated as an essential component in the unified picture of AGN, and is key to the understanding of the nature of these objects.

Past work on the X-ray spectrum of NGC 3783 has yielded information about the warm absorber ionization, but has not provided detailed information or constraints on the gas dynamics. Fitting of photoionization models to the NGC 3783 Chandra spectrum requires at least two components with different ionization parameters in order to fit the line strengths (Kaspi et al. 2002; Krongold et al. 2003). There is evidence for kinematic differences between these components, but they are not apparent in all the lines. There may exist a dynamical relationship between the UV and X-ray absorbers. In NGC 3783 some of the velocity components seen in the UV are detectable in the X-ray lines, but not all (Crenshaw et al. 1999). There is no clear correlation between the velocity of the gas taken from X-ray measurements with the quantities associated with the ionization conditions in the gas such as the ionization potential of the ions (Kaspi et al. 2002) or ionization parameter. The picture that emerges is of an outflow with typical velocity $500-1000 \mathrm{~km} / \mathrm{s}$, but no clear evidence for the acceleration to these velocities, or about the geometry or driving mechanism for the flow.

The $850 \mathrm{ksec}$ Chandra observation of NGC 3783 is likely to remain among the most detailed and best-exposed spectrum of an AGN warm absorber for some time to come. Thus, some effort is justified in extracting the maximum information from it, and that is the main goal of the work we report in this paper. In doing so, we attempt to make use of a slightly different type of information contained in the spectrum than has been previously considered. Analysis efforts so far have focussed on understanding the profile of the absorption lines in this spectrum in terms of line centroids, strengths, and widths in both X-ray (e.g. Kaspi 
et al. 2002; Krongold et al. 2003) and UV (e.g. Crenshaw et al. 1999; Gabel et al. 2003a). Kaspi et al. (2002) reported that most of the absorption lines in the X-ray spectrum of NGC 3783 show asymmetry, having more extended blue wings than red wings. However no tool has been developed to quantify this asymmetry line-by-line. One hypothesis which has been proposed to explain the asymmetries of the X-ray line profiles is that we are observing two or more velocity components blended, with column densities smaller at larger velocity outflow (e.g. for the O viI lines). Support for this idea comes from the observation of multiple discrete components in the UV lines, some of which share outflow velocities with the X-ray lines. Recent observations show evidences of a correspondence between the UV velocity components and the X-rays (e.g., Krongold et al. 2003; Netzer et al. 2003; Krongold et al. 2005). Although Krongold et al. (2003) and Netzer et al. (2003) among others, demonstrate that the inner shell transitions of Fe VII-XII seen in the X-ray region are formed by conditions that favor the UV lines production, this is progressively less evident for X-ray lines coming from higher ionization states. Furthermore, the cross sections for the UV lines are greater than those for X-ray lines by factors $\gtrsim 10^{3}$, so that discrete components appearing in UV lines could result from very small fractional changes in the flow properties. Finally, it appears that outflows from objects within our galaxy, such as hot stars, are characterised by essentially monotonic acceleration from subsonic speed to a speed which is characteristic of the escape velocity from the star. For these reasons, in this paper we will explore the hypothesis that the flow we observe in the X-ray band can be characterized as smooth and monotonically accelerated. This does not preclude the existence of discrete absorption components which affect the UV line profiles, but implicitly assumes that they are small perturbations on an essentially smooth flow.

In this paper we present fits of the profiles of the strongest lines in the NGC 3783 HETG spectrum using a parametrization technique similar to that used in profile fits in hot stars. This has the advantage that we can examine the implications of the fits for the global properties of the flow. There is clear evidence for asymmetry in some lines, and the line shapes contain information which can be interpreted in terms of the velocity law in the flow, the mass budget, and the ionization balance. We also find evidence for a systematic dependence of the profile shapes on the properties of the parent ion, and this has implications for the dynamics of the gas in the outflow from NCG 3783.

\section{Observations and data reduction}

The $900 \mathrm{ks}$ spectrum of the Seyfert 1 Galaxy NGC 3783 used in this work is composed of six smaller observations, one of them reported by Kaspi et al. (2001) with a total $56 \mathrm{ks}$ 
of exposure time and the other five made in a large X-ray campaign (Kaspi et al. 2002) with $\sim 170 \mathrm{ks}$ each (see Table 1). We have analyzed all of these data uniformly using the most recent versions of the Chandra Interactive Analysis of Observations software (CIAO 3.0) and the matrix response calibrations (CALDV V.2.3) available to us to build new ancillary response files (ARF files). We extracted the negative and positive first order spectra of the MEG (Medium Energy Grating) and HEG (High Energy Grating). These have approximately $0.023 \AA$ and $0.012 \AA$ spectral resolution, respectively. In the energy range $0.5-10 \mathrm{keV}$, the total numbers of counts in the first order is 583,196 for the MEG and 313,861 for the HEG. The S/N values at $\sim 7 \AA$ are $\sim 23$ for the MEG and $\sim 10$ for the HEG in the combined $900 \mathrm{ks}$ spectra (Kaspi et al. 2002) using the default CIAO bins of $0.005 \AA$ and $0.0025 \AA$ respectively. We have checked all our measurements against the HEG data and find no significant differences. Moreover, we find the MEG data better because of the better signal to noise ratio in the spectral region of interest, and so in what follows we present results based on the analysis of the added negative and positive first-order MEG data. A detailed discussion of systematic uncertainties in wavelengths and flux measurements is presented by Kaspi et al. (2001), Kaspi et al. (2002) and references therein. Kaspi et al. (2002) reported a negligible background level with HEGTS/ACIS $\lesssim 0.5 \%$ of the signal. For this reason we did not subtract any background in this survey. In the analysis of the following sections we adopt single bin resolution elements, the default MEG size $(0.005 \AA)$, giving us the maximum grating resolution.

\subsection{Fitting of the Continuum}

The continuum of NGC 3783 in the range $0.5-10 \mathrm{keV}$ has been fitted by a power law with a photon index that spans from $\Gamma \sim 1.5$ to $\Gamma \sim 1.83$ (Blustin et al. 2002; De Rosa et al. 2002), sometimes including a thermal component with $k T \sim 0.1 \mathrm{keV}$ (Krongold et al. 2003). Such fits depend on disentangling the true continuum from the effects of the absorption lines, which may include many lines which are unresolved and overlapping, and from the effects of possible emission. An accurate analysis of this type requires a model capable of fitting essentially all the absorption and emission features in the spectrum, including those which cannot be clearly resolved. Current spectral models have been very successful in fitting the majority of the strong lines (e.g. Krongold et al. 2003; Netzer et al. 2003), but currently available databases of atomic lines are insufficient to allow for highly accurate fits to the global spectrum (including weak features in both absorption and emission). For this reason, in our analysis we do not attempt to fit the global continuum. Instead, we work with distinct narrow spectral bands $(\simeq 1 \AA)$ centered on the lines of interest. In each band we fit the spectrum to the line profile described below, plus a local continuum which is a power law 
with $\Gamma=1.77$ and normalization adjusted to each narrow band.

In Figure 2 we show the continuum level linked together over the entire spectral range. In spite of the uncertainty associated with the continuum placement, we are confident that our choice of continuum does not introduce major errors into the fits of the absorption line profiles. This is because our continuum is a good fit to the local spectrum surrounding each of the strong lines, and because we have chosen our fitting intervals in such a way as to have the lines in the middle of each interval. This avoids dependence of the derived line properties on the continuum near the band boundaries, where there are unphysical discontinuities every $\sim 0.6 \AA$, from the connections between some of the narrow bands. Nonetheless we take into account possible variations of up to $10 \%$ in the continuum level in our analysis of errors, as described in the next section.

\subsection{Line Identification and Atomic Data}

The spectrum of NGC 3783 contains a large number of absorption lines that are clearly apparent as local minims. Kaspi et al. (2002) identify 135 of them. In addition, the apparent bumpiness of the continuum suggests that there may be more weak or blended unidentified features. These complicate the fitting of the continuum which is reminiscent of the many emission lines of Fe II that affect the fitting of continuum spectra of AGN and some stars in the UV (Verner et al. 2003). In order to minimize the effects of blending and to allow for accurate determination of the outflow, we choose a subset of strong resonance lines with no obvious signs of blending (see Table 2), and whose wavelengths are accurately known (within $\simeq 0.01 \AA$ ) from experiment (e.g. Brown et al. 2002, and NIST ${ }^{1}$ ). These lines were also identified by Kaspi et al. (2002) (see Table 3 of Kaspi et al. 2002).

\subsection{Line profiles}

In Figures 3-5 we show examples of several regions of the spectrum containing absorption lines. Several of these are clearly asymmetric. In the case of O VIII $\lambda 19 \AA$ there is also evidence for an emission component, reminiscent of $\mathrm{P}$ Cygni profiles. There is a few other lines in the spectrum which share this property, but in the great majority the emission is weak or absent. The goal of our analysis is to characterize and understand the dynamics of the gas flow from the shape of these lines, and in doing so we concentrate exclusively on

\footnotetext{
${ }^{1}$ http://www.nist.gov
} 
the absorption part. This is because the emission appears to be coming only from a small subset of the lines, and because the emission component in O VIII $\lambda 19 \AA$ is narrower than the absorption component, so that the absorption at high Doppler velocities is safely out of the wings of the emission line. This approach has been shown to be fruitful in studies of the profiles in hot stars, for which the modeling of the emission component is less reliable than the study of the absorption (Lamers et al. 1987). Possible reasons for this include the contributions of thermal emission to the emission component, poorer understanding of atomic emission rates, and the fact that the emission depends more sensitively on the global properties of the flow geometry than does absorption. In AGNs it is likely that the flows are not spherically symmetric which will add further uncertainty to any theoretical prediction of the emission/absorption ratio. This provides further motivation to our decision to limit the study to solely absorption.

Our procedure for analysis of the line profiles consists of fitting the flux in each narrow $1 \AA$ band containing one of the lines of interest to a model of the form

$$
F(\lambda)=F_{\text {cont }}(\lambda) \times \exp [-\tau(w)]
$$

where $F_{\text {cont }}(\lambda)$ is the continumm flux described in the previous subsection.

Our wind model is based on the Castor \& Lamers (1979) P Cygni profile atlas, built using the Sobolev approximation and parametrized by two functions, the velocity law $v(r)$ and the optical depth as function of the velocity $\tau(v)$. Then Lamers et al. (1987) developed the SEI method (Sobolev source function with Exact Integration) and applied to compute mass loss rates and terminal velocities in winds of Wolf-Rayet stars, being as accurate as the comoving-frame method of Mihalas et al. (1975) but much more numerically efficient to use interactively in the fitting of calculated profiles to the observations. In the Sobolev approximation the radial optical depth is (Castor et al. 1975)

$$
\tau_{s o b}(v)=\frac{\pi e^{2}}{m c}(g f)_{l u}\left(\frac{n_{l}}{g_{l}}-\frac{n_{u}}{g_{u}}\right) \lambda_{0}\left(\frac{d v}{d r}\right)^{-1}
$$

where $f$ is the absorption oscillator strength, $\lambda_{0}$ (in $\mathrm{cm}$ ) is the laboratory wavelength of the transition, $n_{l}, n_{u}$ (in $\mathrm{cm}^{-3}$ ), $g_{l}, g_{u}$ are the number densities and the statistical weights of the lower and upper levels of the transition respectively, and $d v / d r$ is the velocity gradient in the wind.

For resonance lines $\left(n_{u} / g_{u} \ll n_{l} / g_{l}\right)$ one can approximate

$$
\tau_{s o b}(v) \approx \frac{\pi e^{2}}{m c} f \lambda_{0} n_{l}\left(\frac{d v}{d r}\right)^{-1}
$$


The velocity law used throughout this work is based on the stellar wind theory of Castor et al. (1975), where the wind is assumed to be driven by the radiative force on spectral lines and its velocity varies with the distance as

$$
w(x)=w_{0}+\left(1-w_{0}\right)\left(1-\frac{1}{x}\right)^{\beta},
$$

here $w$ is the velocity normalized to the terminal velocity of the wind $v_{\infty}, w_{0}$ is the velocity in the base of the wind and $x$ is the distance normalized to the radius of the central core $r / r_{0}$. The parameter $\beta$ is the quantity governing the slope of the velocity with the distance and its ad hoc value depends on the type of radiative force acting on the wind. Analysis of hot stars suggests that $0.5 \leq \beta \leq 1$, and we adopt the value $\beta=1$ (Lamers et al. 1987).

¿From this, the line optical depth can be parametrized as in Lamers et al. (1987):

$$
\tau(w)=\left(T_{\text {tot }} / I\right)\left(w / w_{1}\right)^{\alpha_{1}}\left(1-\left(w / w_{1}\right)^{1 / \beta}\right)^{\alpha_{2}}
$$

where $w=v / v_{\infty}=\left(c / v_{\infty}\right)\left(1-\lambda / \lambda_{0}\right)$. In this equation we use $w_{1}=v_{1} / v_{\infty}$ where $v_{1}$ is the position of the blue edge of the line in velocity space (see, e.g., Lamers \& Morton 1976). The total optical depth is

$$
T_{\text {tot }}=\left(\pi e^{2} / m_{e} c\right) f \lambda_{0} N_{i} / v_{1},
$$

where $N_{i}$ is the total column density of the absorbing ion, and

$$
I=w_{1} \int_{0}^{1} y^{\alpha_{1}}\left(1-y^{1 / \beta}\right)^{\alpha_{2}} d y
$$

is the normalization integral.

It is important to note that although equation (5) was derived for radiative outflows, it is a convenient parametric formula for driving mechanisms as well, in which case the free parameters $\alpha_{1}, \alpha_{2}$, and $\beta$ may adopt slightly different physical meanings.

In order to illustrate the role of each parameter on the line profile, we show in Figure 1 the results of various models. In Figure 1(b), we can see the sensitivity of the profile when $\alpha_{2} / \alpha_{1}>1$ (blue lines) for different values of $\alpha_{2}$. Figure $1(\mathrm{c})$ shows the case when a line is symmetric, i.e. $\alpha_{2} / \alpha_{1}=1$, and Figure $1(\mathrm{~d})$ shows lines with $\alpha_{2} / \alpha_{1}<<1$ (red lines). When $\alpha_{2} / \alpha_{1}$ is large then the line has what we will call 'blue skew', that is the red edge is sharp and the blue wing is more gradual. In this case the slope of the blue wing is controlled by $\alpha_{2}$ (with $\alpha_{1}$ fixed). When $\alpha_{2} / \alpha_{1}$ is small then the line has what we will call 'red skew', that is the blue edge is sharp and the red wing is more gradual. In this case the slope of the red wing is controlled by $\alpha_{1}$ (with $\alpha_{2}$ fixed). This shows that the line profiles can be easily characterized by the value of $\alpha_{2} / \alpha_{1}$. 
Fitting of lines in the spectrum of NGC 3783 was carried out with the Interactive Spectral Interpretation System package (ISIS ver. 1.1.8) using the XSPEC modules from the LHEASOFT libraries. In doing this we constructed a new XSPEC local-module named XSPCYG which is based on the computation of the optical depth as given by equation (5) (Lamers et al. 1987).

Each line fit depends on four free parameters and one fixed; $E_{\text {obs }}, v_{1}, \alpha_{1}, \alpha_{2}$ and $T_{\text {tot }}$. $E_{\text {obs }}$, is the energy of the red edge of the line (in $\mathrm{keV}$ ), which we take as the line transition energy in the rest frame of the galaxy. In Table 3 we present the results of the measurements. In the first and second column we have the ion and the rest wavelength of the line respectively. In the third column we give $v_{1}$, which is the position of the blue edge of each line (in $\mathrm{km} / \mathrm{s}$ ). The parameters describing the asymmetry of the line, $\alpha_{1}$ and $\alpha_{2}$, are in columns fourth and fifth. The integrated optical depth $T_{\text {tot }}$ (eq. [6]) is in the sixth column. Our determinations of the goodness of the fits were carried out computing a local $\chi_{\nu}^{2}$. In column seventh and eighth we give the number of bins included and our calculation of $\chi_{\nu}^{2}$. After finding the best-fit parameters we compute the $90 \%$ of confidence level of all the parameters with the CONF command in ISIS. We included $10 \%$ on the variation of the continuum (through the normalization parameter) and $v_{1}$ in the Monte Carlo searching, to take into account systematic uncertainties in the computation of the confidence intervals. We show in Table 3 the limits associated to each fit parameter. These errors propagate as

$$
\Delta\left(\frac{\alpha_{1}}{\alpha_{2}}\right)=\frac{1}{\alpha_{2}} \Delta \alpha_{1}-\frac{\alpha_{1}}{\alpha_{2}^{2}} \Delta \alpha_{2}
$$

where $\Delta \alpha_{1}=\alpha_{1}(\max )-\alpha_{1}(\min ), \Delta \alpha_{2}=\alpha_{2}(\max )-\alpha_{2}(\min )$, and

$$
\Delta\left(\frac{\bar{w}}{w_{1}}\right)=\frac{1}{w_{1}} \Delta \bar{w}-\frac{\bar{w}}{w_{1}^{2}} \Delta w_{1}
$$

where $\Delta \bar{w}$ is a third of the bin size $\left(0.005 /\left(3 \lambda_{0}\right) c \mathrm{~km} / \mathrm{s}\right)$.

In Figures 3, 4 and 5 we present the fits of three Ly $\alpha$ lines from H-like ions, which sample a range of ionization parameters, i.e. S XVI $\lambda 4.729$, Ne $x \lambda 12.134$, and O VIII $\lambda 18.869$. Two vertical dashed lines are drawn in these figures indicating the zero velocity point, i.e. the rest wavelength in the AGN frame, and where $\bar{w} / w_{1}=1 / 2$ (i.e. where a perfectly symmetric line should have $\alpha_{1}=\alpha_{2}$ ). These figures are representative of the types of parameters which are found to fit the observed lines. They fall into two categories: (1) lines, like S XVI $\lambda 4.73 \AA$ and Ne x $\lambda 12.1 \AA$ (Figs. 3 and 4), which have $\alpha_{2} \simeq \alpha_{1}$, and (2) lines, like $\mathrm{O}$ VIII $\lambda 19 \AA$, which have $\alpha_{2} \gg \alpha_{1}$. The former are symmetric, or nearly so, and the latter are asymmetric, with a pronounced 'blue skew'. In the case of O VIII $\lambda 19 \AA$, this line is rare in the sense it exhibit a clear emission component that accompanies the absorption. while for the vast majority of other lines the emission component, if present, is below the 
noise level of the spectrum. Nonetheless, we use this line to try to estimate the errors introduced into the absorption line fits when emission component is ignored. To this end we include a Gaussian profile to mimic the emission component and obtain the best fit with the set of parameters: AREA $=1.50 \times 10^{-5}+4.34 \times 10^{-6}$ photons $/ \mathrm{s} / \mathrm{cm}^{2}$, CENTER $=19.147_{-0.004}^{+0.003} \AA$ ${ }^{2}$, SIGMA $=9.26 \times 10_{-5.65 \times 10^{-3}}^{-3} \AA$. In the worse case this emission has the effect of blueshifting the minimum point of the absorption component by $\sim 200 \mathrm{~km} / \mathrm{s}$ (from $\sim 780 \mathrm{~km} / \mathrm{s}$ to $\sim 980 \mathrm{~km} / \mathrm{s}$ ) and changes the $\alpha_{1}$ parameter by up to $\sim 20 \%$, while $\alpha_{2}$ that is mostly effected by the blue wing is affected much less. These $\alpha$ parameter change in the sense of adding a red skew to the line; on the other hand, most of the low ionization lines like O VIII exhibit blue skew. Thus, we conclude that even in the likelihood of emission components below the noise level in other lines in the spectrum, the overall results of the present analysis will be substantially unaffected.

\section{Analysis of line fits and the wind properties}

In the previous section we were able to fit the absorption lines in the spectrum based on model line profiles. In this section we check the distribution of the fits in the parameter space of $\alpha_{1}, \alpha_{2}$, and $w$ to explore systematic errors. Next, we study the relationship between the ionization parameters related to the absorption lines and the bulk flow velocity. This allows us to derive conclusions about the physical conditions of the gas flow in NGC 3783 .

\subsection{Line asymmetry distribution}

The model that we have adopted for fitting the lines imposes an important requirement on the distribution of symmetry parameters $\alpha_{1}$ and $\alpha_{2}$ on the velocity space. This condition is derived by finding the points of maximum absorption of every line, i.e. the minims of the absorption profiles, computed as

$$
\left(\frac{d \tau}{d w}\right)_{\bar{w}}=0
$$

where $\bar{w}$ is evaluated at the minimum of the profile in the observed spectra. It is important to point out that $\bar{w}$ is obtained directly from the spectra independently of line fits and the choice of the continuum levels.

\footnotetext{
${ }^{2}$ Is the observed wavelength.
} 
¿From the line optical depth given by equation (5) we get

$$
\left(\frac{\bar{w}}{w_{1}}\right)^{1 / \beta}=\frac{\alpha_{1} / \alpha_{2} \beta}{1+\alpha_{1} / \alpha_{2} \beta} .
$$

This provides a test on the consistency of the model and the line fits since it relates parameters derived from the fits for each line independently, i.e. $\alpha_{1}$ and $\alpha_{2}$, to parameters directly measured from the spectra without any fitting, i.e. $\bar{w}$. In Figure 6 we plot the values of $\left(\bar{w} / w_{1}\right)^{1 / \beta}$ with $\beta=1$ vs. $\alpha_{1} / \alpha_{2}$ for the lines considered in this work. For this plot, the error bars in $\bar{w}$ are taken as $1 / 3$ of the bin size $\left(0.005 / \lambda_{0} \mathrm{c} \mathrm{km} / \mathrm{s}\right)$ and the errors in $\alpha_{1} / \alpha_{2}$ are derived from the fits shown in Table 3 and propagated as in equation (8). We find good agreement between the observed points and the behavior predicted by equation (11) indicated by the solid line in the plot. The computed correlation coefficient is $r=0.98$ and considering weighted points $\chi^{2}=34.63$. Another important conclusion is that approximately $90 \%$ of the points are in the range $\ddot{w} / w_{1} \leq 0.5$ (i.e. $\alpha_{1} / \alpha_{2} \leq 1$ ). These blue lines are all formed in a region of the wind with velocities less than half the velocity reflected by the blue edge, $v_{1} / 2$. These appear as the lines with the greatest 'blue skew', i.e. $\alpha_{2}>\alpha_{1}$. Only a few lines coming from similar ionization state (i.e. $12 \lesssim \xi \lesssim 50$ ) show the red skew character, e.g. Mg XI, Ne IX, and Fe XVII (see Table 3), where the uncertainty due to emission can be greater because of the ratio $\alpha_{1} / \alpha_{2}>1$.

\subsection{The physical conditions in the warm absorber flow}

In order to consider the implications of the correlation shown in Figure 6 between $\left(\bar{w} / w_{1}\right)^{1 / \beta}$ and $\alpha_{1} / \alpha_{2}$, it is useful to discuss the relationship between the values of the parameters which we derive in Table 3 and the parameters which describe the physical conditions in an AGN outflow.

A simple and illustrative scenario for the origin of the warm absorber flow is a spherical wind centered on the source of continuum radiation where the continuum source provides both the illumination and the ionization of the gas in the flow.

We describe the spatial position in the flow by the radius which we denote as $r$, and which we normalize to a scaled variable $x=r / r_{0}$ according to a fiducial position $r_{0}$. The primary observationally derived quantity is the resonance line optical depth which can be written in the Sobolev approximation (eq.[2]) as (Castor et al. 1975)

$$
\tau_{\mathrm{rad}}(v)=\frac{\pi e^{2}}{m c} f \lambda_{0} n_{i}(r)\left(\frac{d v}{d r}\right)^{-1}
$$


where $f$ is the absorption oscillator strength, $\lambda_{0}$ is the laboratory wavelength with $n_{i}$ the number density of the absorbing ions, and $d v / d r$ is the velocity gradient in the wind.

There are two functions responsible for the variation of the optical depth with velocity: the velocity gradient and the ion density. The former is computed from the velocity law given by equation (4). The ion density is given by

$$
n_{i}(x)=A_{E} \times n_{H}(x) \times q_{i},
$$

where $A_{E}=n_{E} / n_{H}$ is the abundance of the element with respect to $\mathrm{H}, q_{i}=n_{i} / n_{E}$ is the ionization fraction, and $n_{H}(x)$ is the gas number density

$$
n_{H}(x)=n_{0} x^{-2} w^{-1}
$$

and

$$
n_{0}=\left(\frac{\dot{M}}{4 \pi \mu m_{H}} r_{0}^{-2} v_{\infty}^{-1}\right)
$$

where $\dot{M}$ is the mass loss rate and $\mu m_{H}$ is the average mass of the particles. At this point we need to adopt a model for the ionization balance. We assume that the flow is in photoionization equilibrium so that the ion fraction at every point depends only on the ionization parameter $\xi$ (the ratio of the ionizing flux $F$ to the gas density $n_{H}$ ). Our definition of $\xi$ is taken from Tarter et al. (1969)

$$
\xi=\frac{4 \pi F}{n_{H}},
$$

and $F=F_{0} x^{-2}$. By combining equations (16), and (14)

$$
\xi=\xi_{0} w,
$$

where $\xi_{0}=4 \pi F_{0} / n_{0}$. Finally we propose an expression for the ion fraction in the wind, based on the assumption that the ion fraction depends as a power law in the ionization parameter

$$
q_{i} \approx q_{0}(\xi / \bar{\xi})^{\eta},
$$

where $\bar{\xi}$ is the ionization parameter where $q_{i}$ is maximum, and $q_{0} \sim 1$ is the maximum ionization fraction. Then the optical depth can be written

$$
\tau_{\text {rad }}(w)=\frac{\pi e^{2}}{m c} f \lambda_{0} n_{0} \frac{r_{0}}{v_{\infty}} q_{0} A_{E} w^{\eta+1 / \beta-2} .
$$

Comparing with equation. (12) for the optical depth, this expression predicts a profile with $\alpha_{2}=0$ and $\alpha_{1}=\eta+1 / \beta-2$. If $\alpha_{1}>0$ such a profile would had a very pronounced 'red 
skew'. This is in conflict with the results of our fits shown in Table 3 where we find $\alpha_{2}>$ $\alpha_{1}$ and $\alpha_{1}>0 . \alpha_{2}=0$ is ruled out for all the lines at the $2 \sigma$ level, and for many at much greater level of confidence. An optical depth of this form also does not address the question of the mass budget, total optical depth, or the driving mechanism of the flow.

The spherical outflow model likely provides an illustration of some of the physical effects important in a warm absorber flow, but clearly does not account accurately for the line profile shapes observed in NGC 3783. Other evidence for non-spherical flow geometry comes from unified scenarios (Antonucci \& Miller 1985) in which the gas $\sim 1$ pc from the AGN center is concentrated in a torus which blocks a direct view of the center. If the warm absorber flow originates from the torus, as suggested by Krolik \& Kriss (2001) and Chelouche \& Netzer (2003) then it may have axial symmetry, but likely will not resemble a spherically symmetric flow close to its origin. Similar arguments apply if the flow originates from an accretion disk, as has been suggested for broad absorption line quasar outflows (e.g. Murray et al. 1995). In either case it is likely that the flow divergence does not obey $r^{-2}$ dilution due to collimation by a confining medium, distributed sources of gas in the flow, magnetic fields, or transition from a planar flow into a spherical flow. Also, the radiation field may not be solely due to a point source which is diluted by geometrically, owing to attenuation or to a source which is spatially extended on a length scale comparable to the flow size.

In an attempt to take these effects into account we modify the model equations as follows. Firstly, we write the density as:

$$
n_{H}(x)=n_{0} x^{-2+\kappa} w^{-1}
$$

where a positive value of $\kappa$ implies that the gas flow dilutes more slowly than in a free spherical expansion, i.e. that there are sources of gas embedded in the flow, or that the flow is confined. A negative value corresponds to sinks of gas in the flow, or expansion of an initially confined flow in a flaring geometry. Secondly, we allow the radiation flux to have a form

$$
F=F_{0} \times x^{-2-p}
$$

where $p$ is an index to mimic a deviation of flux from the pure geometrical dilution case. This is expected if the gas has a significant optical depth (if $\mathrm{p}$ is positive) or if there are sources of radiation embedded in the flow (if $\mathrm{p}$ is negative). Here and in what follows, we set $\beta=1$ for simplicity. Then we find that

$$
\xi=\xi_{0} w \times(1-w)^{\Gamma}
$$

where $\Gamma=p+\kappa$, and then, comparing the resulting expression for line optical depth with equation (12) we find

$$
\alpha_{2}=\Gamma \eta-\kappa=\eta p+\kappa(\eta-1)
$$


and

$$
\alpha_{1}=\eta-1 .
$$

The quantities $p$ and $\kappa$ provide insight into the conditions in the warm absorber flow. The parameter $\eta$ depends on the physics of ionization, and so will vary from one ion to another, according to the differing conditions in the gas. $p$ and $\kappa$ are universal quantities affecting all the lines with comparable weight.

A more detailed discussion requires examination of ionization balance in order to estimate the values for $\eta$. We expect the ion fraction $q_{i}(\xi)$ to be a function with a single maximum at some value of $\xi=\bar{\xi}$, and decreasing monotonically at larger and smaller values. $\eta$ describes the logarithmic slope of the $q_{i}(\xi)$ curve, and it is well known that the absolute value of this slope increases with $|\xi-\bar{\xi}|$. The ionization parameter in the flow is given by equation (22). If $p=\kappa=0$ then $\xi$ is an increasing function of velocity. Otherwise, $\xi$ is a decreasing function of position (corresponding to $1 /(1-w)$ ) at large position. At $w=0.9$ $\xi=\xi_{0} / 10^{p+\kappa}$. Thus, it is likely that $\eta$ is not constant through the flow for most ions.

We will nonetheless consider briefly here a highly simplified scenario in which we assume that $\eta$ is constant for each ion throughout the flow. This is intended to illustrate qualitatively how systematic differences in line asymmetries can arise, not to test a specific dynamical model. Also for the following analysis we assume that the ion number density behaves as a power law with the ionization parameter with positive $\eta$. This is plausible because the maximum absorption depends on the product of $q_{i}$ with the gas density shifting this peak. As result we can make the analysis in the region where $\xi \lesssim \bar{\xi}$, (i.e $\eta>0$ ).

We assume here that the flow has a characteristic ionization parameter $\xi_{0}$ which is much greater than $\bar{\xi}$ for all the ions in the flow. And we will assume that for each ion $\eta$ depends only on the relative values of $\bar{\xi}$ and $\xi_{0}$. Specifically, if $\eta=\left|\log (\bar{\xi})-\log \left(\xi_{0}\right)\right|^{1.5}$ for all ions, and if $\log \left(\xi_{0}\right)=4, p=1$ and $\kappa=0.5$ then we predict $0<\alpha_{1}<4$ and $1<\alpha_{2}<8$. This is crudely consistent with the results in Table 3. Furthermore, the results of in Table 3 show an inverse correlation of both $\alpha_{1}$ and $\alpha_{2}$ with $\log (\bar{\xi})$. Figures 7 and 8 show $\alpha_{1}$ and $\alpha_{2}$ plotted vs. $\log (\bar{\xi})$ as error bars, along with the best fit linear regression lines, plotted as long dashed curves. The values of $\bar{\xi}$ were taken from the ionization balance curves of Kallman \& Bautista (2001). The regression fits were calculated using the weighted least squares algorithm of Akritas \& Bershady (1996), which takes into account the non-uniform errors in finding the best fit. The Pearson's correlation coefficients are -0.49 and -0.55 for $\alpha_{1}$ and $\alpha_{2}$, respectively, versus $\log (\bar{\xi})$. Also shown in Figures 7 and 8, as the short dashed curves, are the values of $\alpha_{1}$ and $\alpha_{2}$ predicted by equations (24) and (23), with the formulation for $\eta$ described above, $p=1$ and $\kappa=0.5$. This shows that such an analytic model is an adequate description of the best fit regression line in both cases. What this shows is that the lines in NGC 3783 never have a 
red skew; they either have a blue skew or are more nearly symmetric. The amount of blue skew is greater for lines with lower $\bar{\xi}$, i.e. lines whose parent ion is most abundant at lower ionization parameter. This can be explained, crudely, if the lines from low $\bar{\xi}$ ions have a greater value of $\eta$, i.e. they are more sensitive to changes in the ionization parameter of the flow, than lines from greater $\bar{\xi}$ ions.

Another way of representing the global properties of the outflow, is by examining the relation between ionization parameter at the absorption line minims vs. the line velocity. From our model such a relation is given by equation (22). In Figure 9 we plot the expected ionization parameter for maximum optical depth of each line vs. the measured reduced velocity at the line minimum, i.e. $\bar{w}$. For this plot we have adopted a final wind velocity $v_{\infty}=1000 \mathrm{~km} / \mathrm{s}$. The figure also shows the curve given by equation (17), with $\beta=1$ and $\Gamma$ and $\xi_{0}$ adjusted through $\chi^{2}$ fitting to the data. The values found for these two parameters are $\Gamma=5.18 \pm 2.28$ and $\xi_{0}=15410 \pm 120$.

The results presented so far in this section can be summarized as follows: A simple spherical flow conserving mass predicts ionization parameter increasing monotonically with velocity. If the ion fraction is a monotonically increasing function of ionization parameter, or even if it decreases more slowly than $1 / \beta-2$, then the line will have a net red skew which is not consistent with the fitted line profiles. Departures from this scenario allow for blue skew since they allow the optical depth to decrease with velocity. This can occur if the ionization parameter decreases at large distance or high velocity owing to additional sources of gas or collimation of the flow, or due to attenuation of the ionizing radiation. The observational result that the blue skew is more pronounced for lines with low values of $\bar{\xi}$ suggests that the ionization fraction is an increasing function of ionization parameter, and that the rate of this increase is greater for ions with low $\bar{\xi}$. That is, these ions are more sensitive to both the increase and decrease in $\xi$ as the wind accelerates.

\section{Summary and discussion}

We have reanalyzed the $900 \mathrm{ks}$ CHANDRA spectrum of NGC 3783 . The purpose of this work was to explore the kinematics of the warm absorber in absence of a linear correlation between the Doppler-shifts of the lines and the ionization energy of the absorbing ion, as noticed previously by Kaspi et al. (2002). We fit to model line profiles similar to the SEI profiles of Lamers et al. (1987), developed to model hot star lines. The line profiles predicted by this model are generally consistent with the observed data and allow us to characterize the main lines in the spectra, in terms of total optical depth, terminal velocity of the wind, and symmetry parameters. 
Our model for the wind also allows us to explain the relationship in the spectrum between the ionization parameter at which the lines absorb and the velocity shifts of the lines. Moreover, a theoretically predicted curve (eq. [22]) can be adjusted to fit the observed data. In doing this we find a value for the exponent $\Gamma \simeq 5$. This exponent results from the sum of two other parameters that represent a deviation of the radiation flux away from the simple geometrical dilution and deviation of the gas density from normal spherical decay under hydrostatic equilibrium. The large value of $\Gamma$ suggests a combination of a decay of the radiation flux with distance from the source and departure from spherical dilution of the gas density.

The model also predicts a relationship between the velocity line shift, measured at the line minimum, and the line symmetry parameters. The observed data agrees well with such a relationship. This is interesting because it involves the line shifts which are measured independently of the fits of the lines and the symmetry parameters for the lines, which depend on the wind properties as well as on the micro physics of the absorbing ions. The results of this work do not directly address the issue of the dynamics of the wind, its origin, or the details of ionization properties. Our results try to provide a framework for testing detailed dynamical models.

The present interpretation about the asymmetry of the absorption lines as being due to a continuum wind flow is different from the interpretation of previous authors who propose that the X-rays might come from the blend of at least three UV components. But these two ideas may not be necessarily contradictory, because a continuum flow that yields a spectrum with line velocity spread between 500 and $2000 \mathrm{~km} / \mathrm{s}$ could well be approximated by the emission of two of three discrete components, within the limitations (in resolution and signal-to-noise) of current spectra. However, the scenario of multiple components raises the question of how are these components related to each other and how the flow maintains the equilibrium. On the other hand, this scenario has the advantage of explaining the apparent kinematic relation between the UV and X-ray spectra. This point is currently under investigation within the wind flow model and will be the subject of a further publication.

We thank the anonymous referee for constructive comments. The observation would not have been possible to analyze without the enormous effort of the Chandra team.

\section{REFERENCES}

Akritas; M. G. \& Bershady, M. A. 1996, ApJ, 470, 706 
Antonucci, R. R. J. \& Miller, J. S. 1985, ApJ, 297, 621

Blustin, A. J., Branduardi-Raymont, G., Behar, E., Kaastra, J. S., Kahn; S. M., Page, M. J., Sako, M., \& Steenbrugge, K. C. 2002, A\&A, 392, 453

Brown, G. V., Beiersdorfer, P., Liedahl, D. A., Widmann, K., Kahn, S. M., \& Clothiaux, E. J. 2002, ApJS, 140, 589

Canizares, C. R., Huenemoerder, D. P., Davis, D. S., Dewey, D., Flanagan, K. A., Houck, J., Markert, T. H., Marshall, H. L., Schattenburg, M. L., Schulz, N. S., Wise, M., Drake, J. J., \& Brickhouse, N. S. 2000, ApJ, 539, L41

Castor, J. I., Abbott, D. C., \& Klein, R. I. 1975, ApJ, 195, 157

Castor, J. I. \& Lamers, H. J. G. L. M. 1979, ApJS, 39, 481

Chelouche, D. \& Netzer, H. 2003, MNRAS, 344, 223

Crenshaw, D. M., Kraemer, S. B., Boggess, A., Maran, S. P., Mushotzky, R. F., \& Wu, C. 1999, ApJ, 516, 750

De Rosa, A., Piro, L., Fiore, F., Grandi, P., Maraschi, L., Matt, G., Nicastro, F., \& Petrucci, P. O. 2002, A\&A, 387, 838

Fabian, A. C., Kunieda, H., Inoue, S., Matsuoka, M., Mihara, T., Miyamoto, S., Otani, C., Ricker, G., Tanaka, Y., Yamauchi, M., \& Yaqoob, T. 1994, PASJ, 46, L59

Gabel, J. R., Crenshaw, D. M., Kraemer, S. B., Brandt, W. N., George, I. M., Hamann, F. W., Kaiser, M. E., Kaspi, S., Kriss, G. A., Mathur, S., Mushotzky, R. F., Nandra, K., Netzer, H., Peterson, B. M., Shields, J. C., Turner, T. J., \& Zheng, W. 2003a, ApJ, 583, 178

-. 2003b, ApJ, 595, 120

George, I. M., Turner, T. J., Netzer, H., Nandra, K., Mushotzky, R. F., \& Yaqoob, T. 1998, ApJS, 114,73

Halpern, J. P. 1984, ApJ, 281, 90

Kallman, T. \& Bautista, M. 2001, ApJS, 133, 221

Kaspi, S., Brandt, W. N., George, I. M., Netzer, H., Crenshaw, D. M., Gabel, J. R., Hamann, F. W., Kaiser, M. E., Koratkar, A., Kraemer, S. B., Kriss, G. A., Mathur, S., Mushotzky, R. F., Nandra, K., Peterson, B. M., Shields, J. C., Turner, T. J., \& Zheng, W. 2002, ApJ, 574, 643 
Kaspi, S., Brandt, W. N., Netzer, H., George, I. M., Chartas, G., Behar, E., Sambruna, R. M., Garmire, G. P., \& Nousek, J. A. 2001, ApJ, 554, 216

Krolik, J. H. \& Kriss, G. A. 2001, ApJ, 561, 684

Krongold, Y., Nicastro, F., Brickhouse, N. S., Elvis, M., Liedahl, D. A., \& Mathur, S. 2003, ApJ, 597, 832

Krongold, Y., Nicastro, F., Elvis, M., Brickhouse, N. S., Mathur, S., \& Zezas, A. 2005, ApJ, 620,165

Lamers, H. J. G. L. M., Cerruti-Sola, M., \& Perinotto, M. 1987, ApJ, 314, 726

Lamers, H. J. G. L. M. \& Morton, D. C. 1976, ApJS, 32, 715

Mihalas, D., Kunasz, P. B., \& Hummer, D. G. 1975, ApJ, 202, 465

Murray, N., Chiang, J., Grossman, S. A., \& Voit, G. M. 1995, ApJ, 451, 498

Nandra, K. \& Pounds, K. A. 1992, Nature, 359, 215

Netzer, H., Kaspi, S., Behar, E., Brandt, W. N., Chelouche, D., George, I. M., Crenshaw, D. M., Gabel, J. R., Hamann, F. W., Kraemer, S. B., Kriss, G. A., Nandra, K., Peterson, B. M., Shields, J. C., \& Turner, T. J. 2003, ApJ, 599, 933

Reynolds, C. S. 1997, MNRAS, 286, 513

Tarter, C. B., Tucker, W. H., \& Salpeter, E. E. 1969, ApJ, 156, 943

Verner, E., Bruhweiler, F., Verner, D., Johansson, S., \& Gull, T. 2003, ApJ, 592, L59 
Table 1. Chandra Observation Log of NGC 3783.

\begin{tabular}{cccr}
\hline \hline Sequence Number & UT Start & UT End & Time (ks) \\
\hline 700045 & 2000 Jan 20, 23.33 & 2000 Jan 21, 16:20 & 56.4 \\
700280 & 2001 Feb 24, 18:45 & 2001 Feb 26, 17:48 & 165.7 \\
700281 & 2001 Feb 27, 09:18 & 2001 Mar 01, 09:10 & 168.8 \\
700282 & 2001 Mar 10, 00:31 & 2001 Mar 11, 23:30 & 165.5 \\
700283 & 2001 Mar 31, 03:36 & 2001 Apr 02, 02:48 & 166.1 \\
700284 & 2001 Jun 26, 09:57 & 2001 Jun 28, 09:10 & 166.2 \\
\hline
\end{tabular}

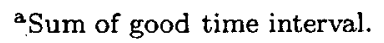

Table 2. Atomic properties of resonance lines in the X-ray spectrum of NGC 3783.

\begin{tabular}{|c|c|c|c|c|}
\hline Ion & wavelength $(\AA)$ & lower level & upper level & f-value ${ }^{a}$ \\
\hline$S X V I$ & 4.729 & $1 s^{2} \mathrm{~S}$ & $2 p^{2} p^{\circ}$ & $4.129 E(-1)$ \\
\hline$S \times V$ & 5.039 & $1 \mathrm{~s}^{2}{ }^{1} \mathrm{~S}$ & $1 \mathrm{~s} 2 \mathrm{p}{ }^{3} \mathrm{p}^{\circ}$ & $7.610 E(-1)$ \\
\hline Si XIII & 5.681 & $1 \mathrm{~s}^{2}{ }^{1} \mathrm{~S}$ & $1 s 3 p^{1} \mathrm{p}^{o}$ & $1.521 E(-1)$ \\
\hline Si XIV & 6.182 & $1 \mathrm{~s}^{2} \mathrm{~S}$ & $2 p^{2} \mathrm{P}^{\circ}$ & $4.136 E(-1)$ \\
\hline Si XIII & 6.648 & $1 \mathrm{~s}^{2}{ }^{1} \mathrm{~S}$ & Is $2 p^{1} \mathrm{P}^{\circ}$ & $7.520 E(-1)$ \\
\hline Mg XII & 7.106 & $1 \mathrm{~s}^{2} \mathrm{~S}$ & $3 p^{2} p^{o}$ & $7.874 E(-2)$ \\
\hline $\mathrm{Mg} \mathrm{XI}$ & 7.473 & $1 \mathrm{~s}^{2}{ }^{1} \mathrm{~S}$ & $1 \mathrm{~s} 4 \mathrm{p}^{1} \mathrm{P}^{\circ}$ & $5.616 E(-2)$ \\
\hline Fe XxilI & 8.303 & $2 s^{2}$ & $2 \mathrm{~s} 4 \mathrm{p}$ & $1.404 E(-1)$ \\
\hline $\mathrm{Mg} X \mathrm{XII}$ & 8.421 & $1 \mathrm{~s}^{2} \mathrm{~S}$ & $2 \mathrm{P}^{2} \mathrm{P}^{\circ}$ & $4.142 E(-1)$ \\
\hline $\mathrm{Mg} \mathrm{XI}$ & 9.169 & $1 \mathrm{~s}^{21} \mathrm{~S}$. & $1 s 2 p^{1} p^{o}$ & $7.383 E(-1)$ \\
\hline Ne $x$ & 9.708 & $1 \mathrm{~s}^{2} \mathrm{~S}$ & $4 \mathrm{p}^{2} \mathrm{p}^{\circ}$ & $2.896 E(-2)$ \\
\hline Ne $x$ & 10.240 & $1 s{ }^{2} \mathrm{~S}$ & $3 p^{2} P^{\circ}$ & $7.883 E(-2)$ \\
\hline Ne IX & 11.547 & $1 \mathrm{~s}^{21} \mathrm{~S}$ & $1 s 3 p{ }^{3} p^{\circ}$ & $1.485 E(-1)$ \\
\hline Fe XXII & 11.770 & $2 s^{2} 2 p$ & $2 s^{2} 3 d$ & $6.444 E(-1)$ \\
\hline $\mathrm{Ne} \mathrm{X}$ & 12.134 & $1 s^{2} \mathrm{~S}$ & $2 p^{2} p^{o}$ & $4.147 E(-1)$ \\
\hline $\mathrm{Fe} \mathrm{XXI}$ & 12.284 & $2 \mathrm{p}^{2}$ & $2 s^{2} 2 p 3 d$ & $1.280 E(+0)$ \\
\hline Fe XviII & 14.373 & $2 s^{2} 2 p^{5}$ & $2 \mathrm{p}^{4}\left(3^{\mathrm{P}}\right) 3 \mathrm{~d}$ & $3.151 E(-1)$ \\
\hline Fe xvin & 14.534 & $2 s^{2} 2 p^{5}$ & $2 p^{4}\left(3^{P}\right) 3 d$ & $2.051 E(-1)$ \\
\hline O VIII & 14.832 & $1 \mathrm{~s}^{2} \mathrm{~S}$ & $5 p^{2} P^{\circ}$ & $1.393 E(-2)$ \\
\hline Fe XvII & 15.015 & $2 \mathrm{p}^{6}$ & $2 p^{5} 3 d$ & $2.676 E(+0)$ \\
\hline O VIII & 15.188 & $1 \mathrm{~s}^{2} \mathrm{~S}$ & $4 p^{2} p^{\circ}$ & $2.896 E(-2)$ \\
\hline O VIII & 16.006 & $1 s^{2} \mathrm{~S}$ & $3 p^{2} P^{o}$ & $7.891 E(-2)$ \\
\hline O VII & 17.200 & $1 \mathrm{~s}^{21} \mathrm{~S}$ & $1 s 6 p^{1} P^{\circ}$ & $1.509 E(-2)$ \\
\hline O vil & 17.396 & $1 s^{21} \mathrm{~S}$ & $1 \mathrm{~s} 5 \mathrm{p}^{1} \mathrm{P}^{\mathrm{o}}$ & $2.679 E(-2)$ \\
\hline O vil & 17.768 & $1 \mathrm{~s}^{21} \mathrm{~S}$ & $1 \mathrm{~s} 4 \mathrm{p}^{\mathrm{I}} \mathrm{P}^{\mathrm{o}}$ & $5.502 E(-2)$ \\
\hline O VII & 18.627 & $1 s^{21} S$ & $1 \mathrm{~s} 3 \mathrm{p}^{1} \mathrm{P}^{\circ}$ & $1.456 E(-1)$ \\
\hline O viII & 18.969 & $1 s^{2} \mathrm{~S}$ & $2 p^{2} P^{\circ}$ & $4.151 E(-1)$ \\
\hline
\end{tabular}

${ }^{a}$ from XSTAR atomic data base 
Table 3. Spectral line fits ${ }^{a}$

\begin{tabular}{|c|c|c|c|c|c|c|c|}
\hline Ion & wavelength $(\AA)$ & $v_{1}(\mathrm{~km} / \mathrm{s})$ & $\alpha_{1}$ & $\alpha_{2}$ & $T_{\text {tot }}$ & \# bins & $\chi_{\nu}^{2}$ \\
\hline S XVI & 4.729 & $2400_{-120}^{+1680}$ & $1.02_{-0.41}^{+1.42}$ & $2.25_{-0.22}^{+4.27}$ & $0.398_{-0.179}^{+0.358}$ & 19 & 0.91 \\
\hline $\mathrm{S} x \mathrm{~V}$ & 5.039 & $2700_{-420}^{+720}$ & $0.90_{-0.36}^{+1.26}$ & $2.55_{-1.40}^{+1.40}$ & $0.342_{-0.159}^{+0.286}$ & 16 & 1.30 \\
\hline Si XIII & 5.681 & $2100_{-840}^{+1470}$ & $0.67_{-0.27}^{+0.93}$ & $1.43_{-0.14}^{+2.40}$ & $0.324_{-0.291}^{+0.1291}$ & 21 & 1.47 \\
\hline Si XIV & 6.182 & $2100_{-1020}^{+600}$ & $0.68_{-0.27}^{+0.25}$ & $2.03_{-0.20}^{+3.185}$ & $0.861_{-0.387}^{+0.775}$ & 26 & 1.42 \\
\hline Si XIII & 6.648 & $1620_{-120}^{+750}$ & $1.06_{-0.42}^{+1.48}$ & $1.54_{-0.84}^{+0.84}$ & $0.739_{-0.335}^{+0.655}$ & 20 & 0.85 \\
\hline $\mathrm{Mg}$ XII & 7.106 & $1680_{-240}^{+480}$ & $1.37_{-0.68}^{+1.92}$ & $2.71_{-1.49}^{+1.04}$ & $0.325_{-0.143}^{+0.150}$ & 11 & 1.39 \\
\hline $\mathrm{Mg} \mathrm{XI}$ & 7.473 & $1260_{-120}^{+360}$ & $0.83_{-0.33}^{+1.16}$ & $0.38_{-0.04}^{+0.72}$ & $0.229_{-0.103}^{+0.103}$ & 12 & 1.33 \\
\hline Fe XXIII & 8.303 & $1500_{-420}^{+90}$ & $0.05_{-0.02}^{+0.08}$ & $1.42_{-0.78}^{+0.78}$ & $0.353_{-0.054}^{+0.317}$ & 15 & 3.26 \\
\hline Mg XII & 8.421 & $1740_{-120}^{+1260}$ & $0.50_{-0.20}^{+0.70}$ & $1.09_{-0.11}^{+2.07}$ & $0.847_{-0.381}^{+0.763}$ & 14 & 1.45 \\
\hline $\mathrm{Mg} X \mathrm{I}$ & 9.169 & $1680_{-240}^{+570}$ & $0.46_{-0.22}^{+0.64}$ & $1.24_{-0.68}^{+0.18}$ & $0.737_{-0.288}^{+0.375}$ & 7 & 3.24 \\
\hline $\mathrm{NeX}$ & 9.708 & $1380_{-100}^{+900}$ & $0.23_{-0.09}^{+0.33}$ & $0.27_{-0.15}^{+0.15}$ & $1.210_{-0.654}^{+0.653}$ & 16 & 2.93 \\
\hline $\mathrm{Ne} X$ & 10.240 & $3780_{-840}^{+540}$ & $1.98_{-0.96}^{+2.75}$ & $10.45_{-5.75}^{+5.15}$ & $0.279_{-0.136}^{+0.208}$ & 27 & 2.88 \\
\hline $\mathrm{Ne} I \mathrm{X}$ & 11.547 & $1380_{-120}^{+600}$ & $2.65_{-1.32}^{+3.71}$ & $1.03_{-0.57}^{+0.57}$ & $0.972_{-0.501}^{+0.875}$ & 13 & 1.92 \\
\hline Fe XXII & 11.770 & $1980_{-240}^{+720}$ & $0.45_{-0.18}^{+0.64}$ & $1.75_{-0.96}^{+0.96}$ & $0.680_{-0.294}^{+0.318}$ & 17 & 0.85 \\
\hline $\operatorname{Ne} x$ & 12.134 & $2940_{-240}^{+120}$ & $2.08_{-0.10}^{+1.98}$ & $5.48_{-3.02}^{+7.95}$ & $0.659_{-0.296}^{+0.296}$ & 25 & 1.43 \\
\hline $\mathrm{Fe} \times \mathrm{XXI}$ & 12.284 & $1800_{-60}^{+330}$ & $0.74_{-0.37}^{+1.04}$ & $1.06_{-0.58}^{+0.58}$ & $1.212_{-0.680}^{+0.550}$ & 16 & 1.35 \\
\hline Fe XvilI & 14.373 & $2340_{-540}^{+60}$ & $1.94_{-0.97}^{+2.72}$ & $\begin{array}{l}5.31+2.92 \\
-2.92\end{array}$ & $0.430_{-0.387}^{+0.078}$ & 25 & 1.41 \\
\hline Fe XVIII & 14.534 & $1140_{-60}^{+600}$ & $2.05_{-0.37}^{+2.87}$ & $2.43_{-1.34}^{+1.34}$ & $1.392_{-0.733}^{+0.826}$ & 14 & 1.88 \\
\hline O vin & 14.832 & $2760_{-660}^{+1170}$ & $4.15_{-2.91}^{+8.26}$ & $14.41_{-5.73}^{+5.73}$ & $0.450_{-0.269}^{+0.033}$ & 29 & 1.28 \\
\hline Fe Xvil & 15.015 & $1020_{-120}^{+510}$ & $8.66_{-1.34}^{+6.12}$ & $1.73_{-0.95}^{+0.95}$ & $4.173_{-3.756}^{+3.756}$ & 11 & 1.05 \\
\hline O VIII & 15.188 & $1980_{-240}^{+690}$ & $0.86_{-0.41}^{+1.21}$ & $1.76_{-0.97}^{+0.97}$ & $0.579_{-0.338}^{+0.212}$ & 22 & 2.51 \\
\hline O VIII & 16.006 & $2160_{-840}^{+600}$ & $3.03_{-1.21}^{+4.24}$ & $8.19_{-0.82}^{+4.56}$ & $0.610_{-0.275}^{+0.549}$ & 27 & 1.45 \\
\hline O VII & 17.200 & $2400_{-480}^{+450}$ & $3.13_{-1.56}^{+4.39}$ & $8.32_{-4.58}^{+4.58}$ & $0.430_{-0.330}^{+0.157}$ & 28 & 2.47 \\
\hline O VII & 17.396 & $2760_{-360}^{+960}$ & $3.15_{-0.20}^{+3.03}$ & $6.61_{-3.64}^{+3.64}$ & $0.601_{-0.342}^{+0.254}$ & 25 & 3.14 \\
\hline O VII & 17.768 & $2280_{-60}^{+1230}$ & $2.17_{-0.02}^{+2.68}$ & $4.80_{-2.64}^{+2.64}$ & $0.678_{-0.385}^{+0.291}$ & 28 & 3.86 \\
\hline O VII & 18.627 & $1800_{-240}^{+630}$ & $3.41_{-1.66}^{+4.78}$ & $6.38_{-3.51}^{+3.51}$ & $0.707_{-0.487}^{+0.040}$ & 13 & 0.86 \\
\hline $\mathrm{O} \mathrm{VIII}$ & 18.969 & $3720_{-480}^{+1320}$ & $1.66_{-0.83}^{+2.32}$ & $8.45_{-4.65}^{+4.65}$ & $0.700_{-0.364}^{+0.434}$ & 44 & 2.07 \\
\hline
\end{tabular}

${ }^{a}$ All uncertainties $90 \%$ of confidence computed with the command conF in Isis 

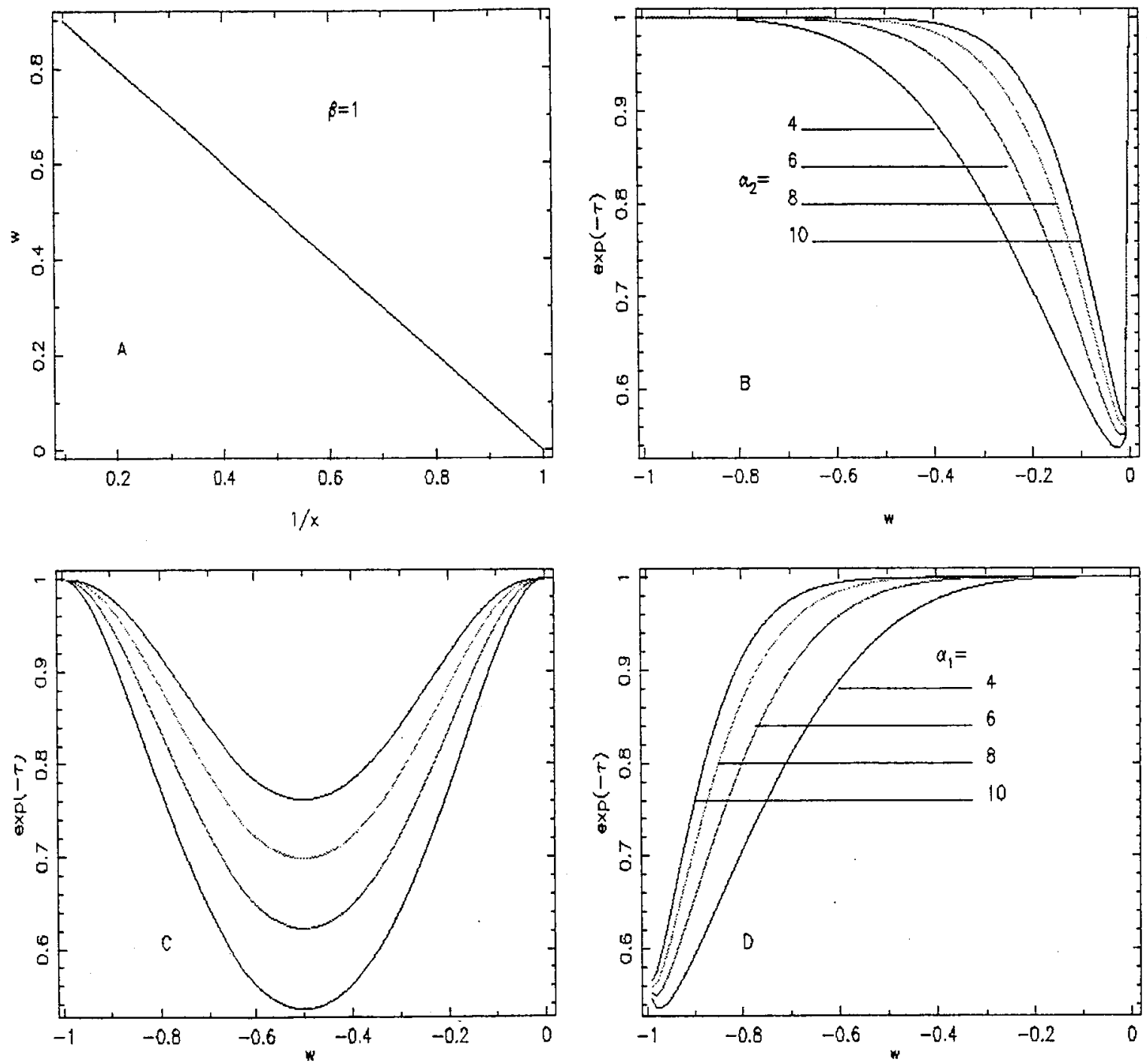

Fig. 1.- Parameter roles: in A) we plotted velocity $w$ vs $1 / x(\mathrm{R} / \mathrm{r})$ with the velocity index $\beta=1$. In B) $T_{\text {tot }} / I=1, \alpha_{1}=0.1$ and the values of $\alpha_{2}$ are labeled. C) shows symmetric profiles with $T_{\text {tot }} / I=10$ and $\alpha_{1}=\alpha_{2}=2$ (black), 2.2 (red), 2.4 (green) and 2.6 (blue). D) $T_{\text {tot }} / I=1, \alpha_{2}=0.1$ and the values of $\alpha_{1}$ are marked. 

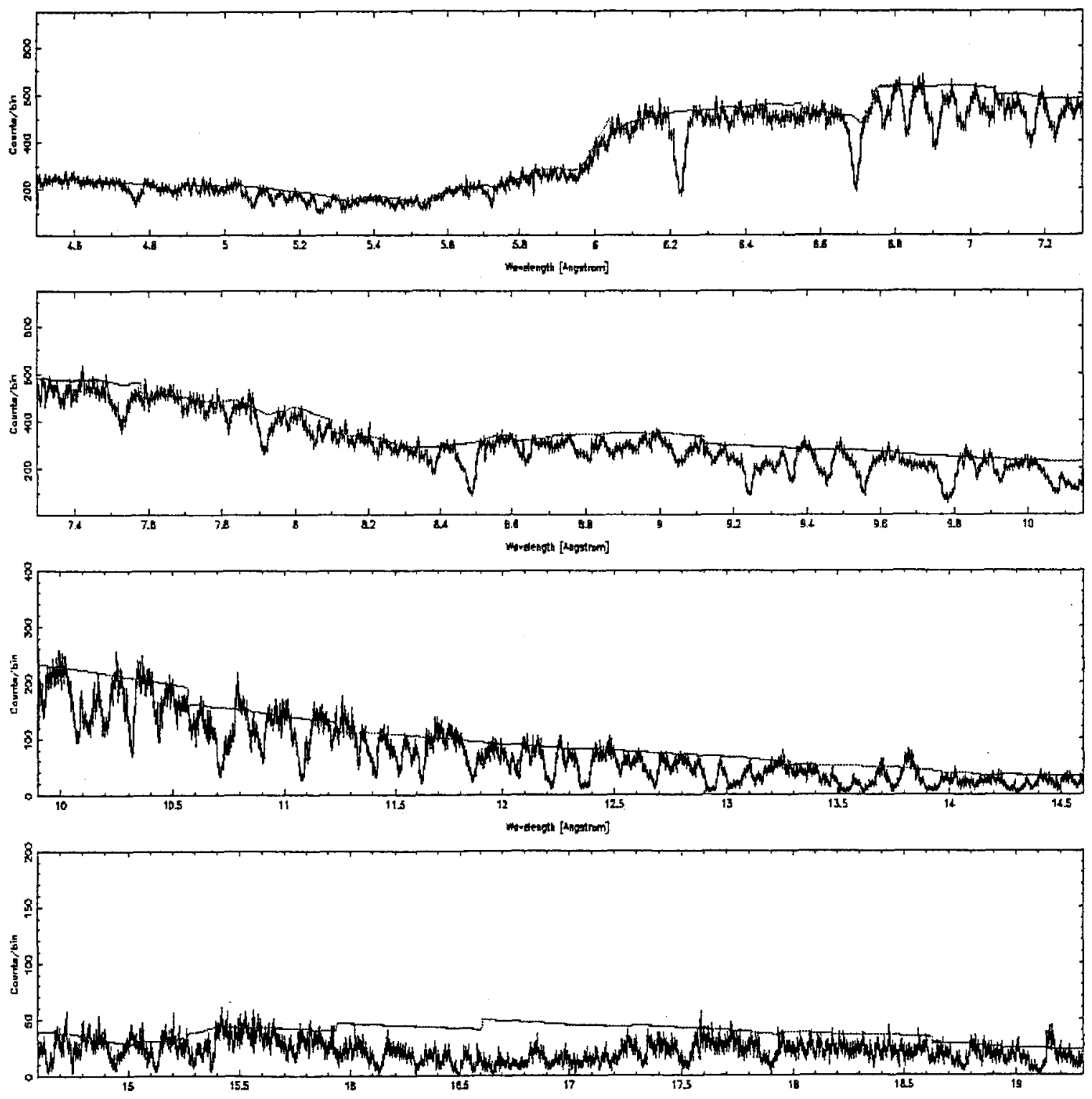

Fig. 2. - 4-20 $\AA$ spectrum of NGC 3783 with the powerlaw continuum used in the fits (in red). 

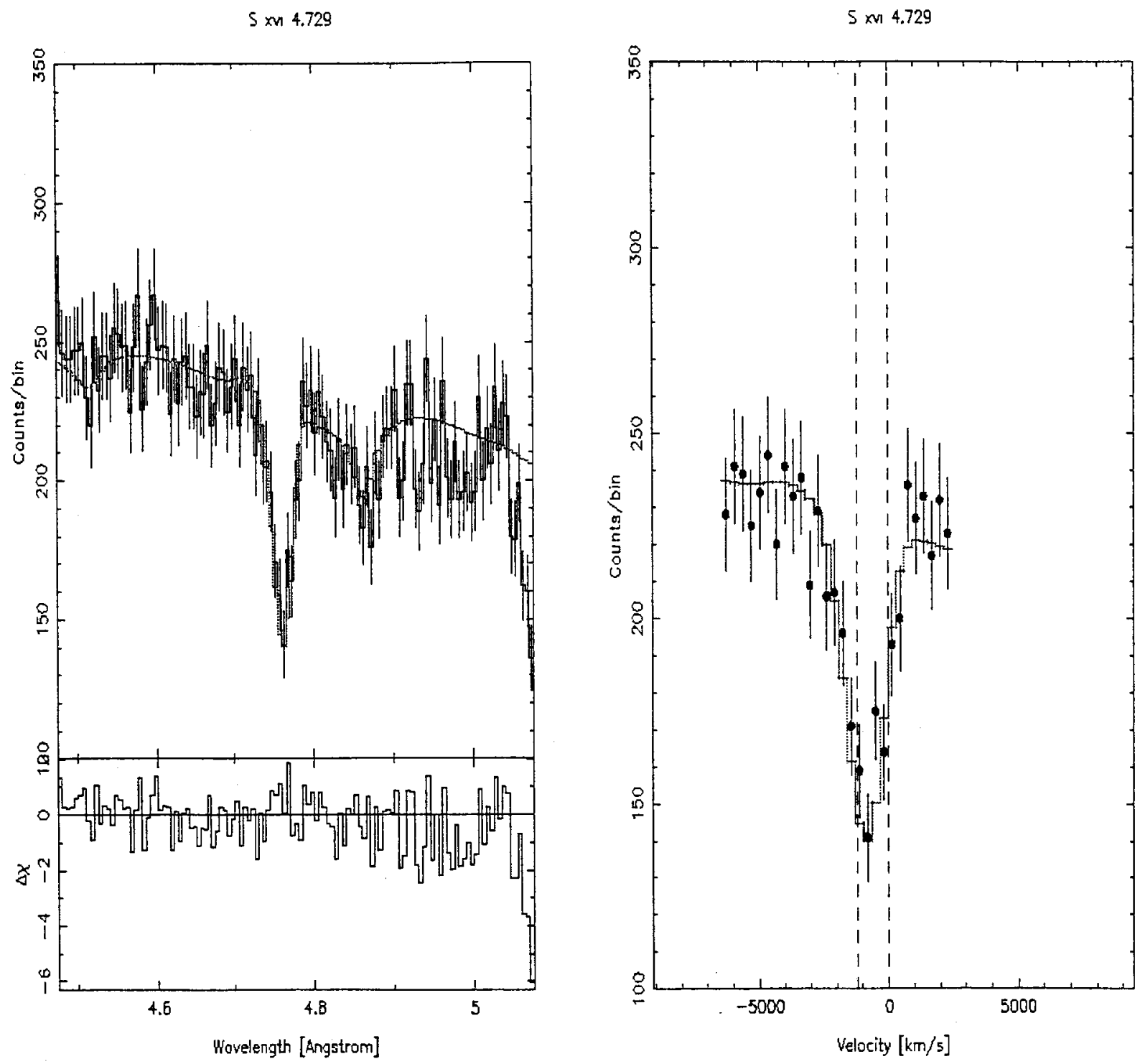

Fig. 3.- Left panel: histogram data of S XVI $\lambda 4.729$ with its adjacencies in the observed wavelength space. The model and the continuum used are superimposed in red. Here a Gaussian has been included (see text for parameter values). Right panel: Velocity spectra with two vertical dashes lines at velocity zero and $1 / 2 w_{1}$. 

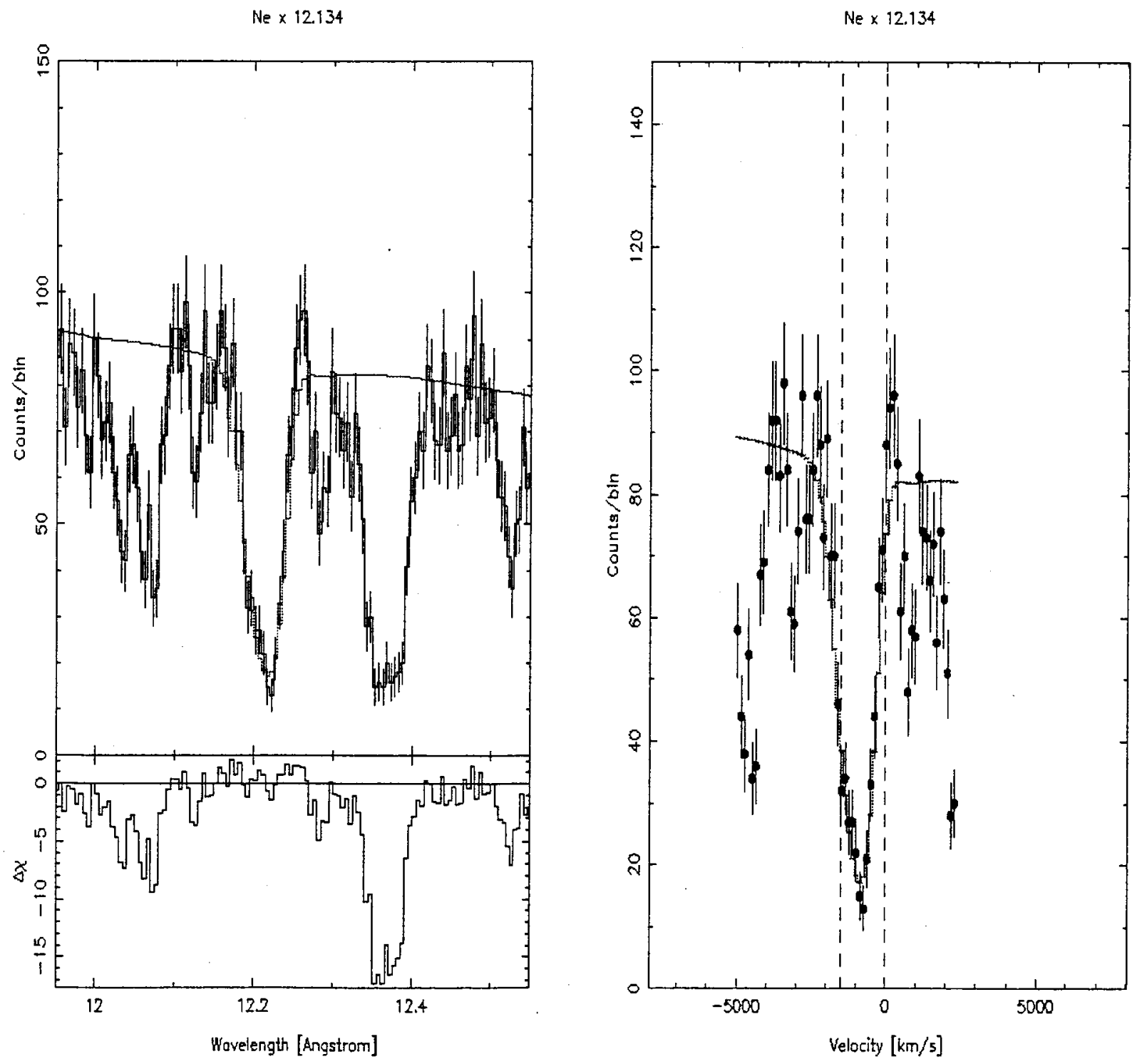

Fig. 4.- Left panel: histogram data of $\mathrm{Ne} \times \lambda 12.134$ with its adjacencies in the observed wavelength space. The model and the continuum used are superimposed in red. Right panel: Velocity spectra with two vertical dashes lines at velocity zero and $1 / 2 w_{1}$. 

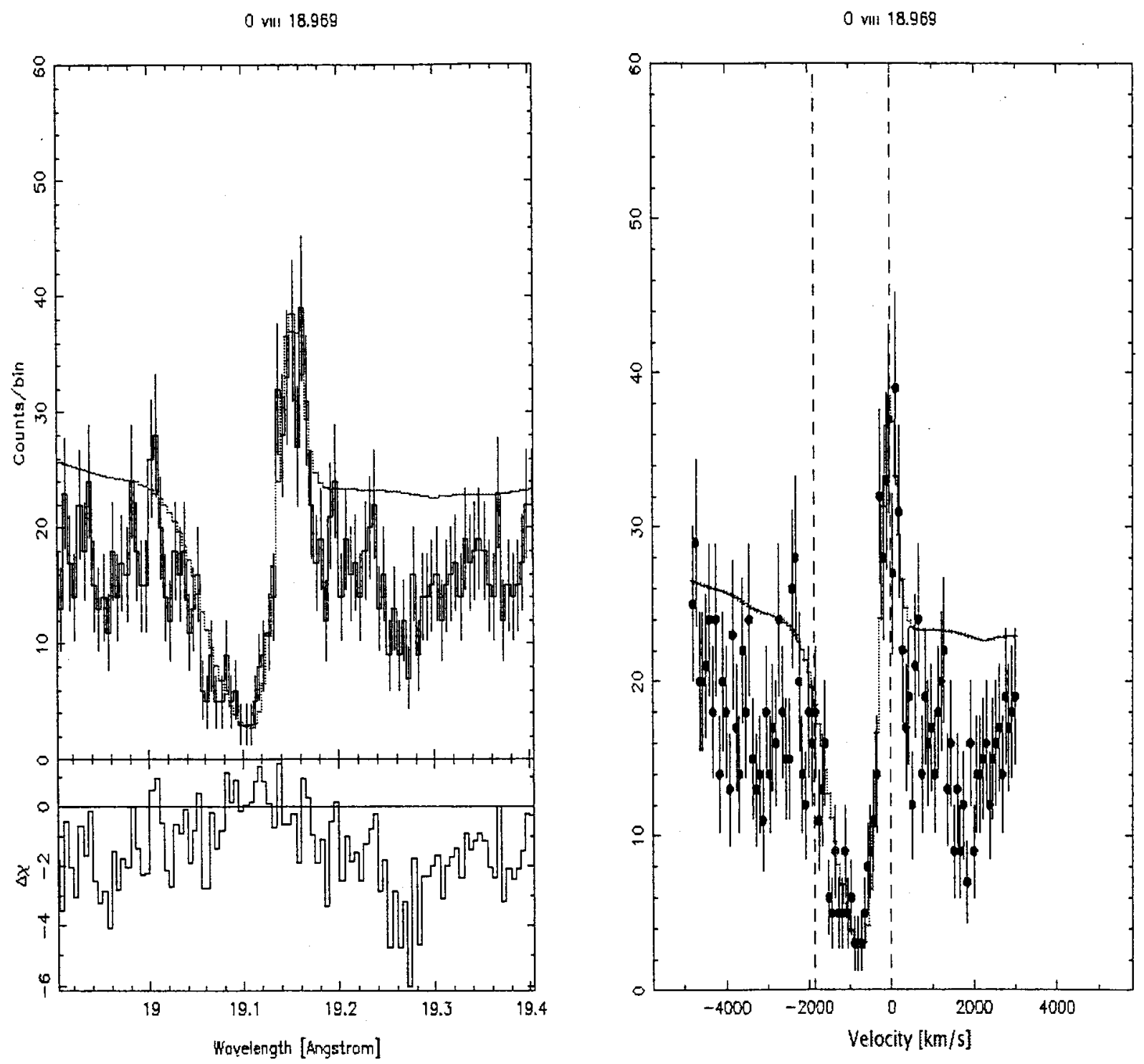

Fig. 5.- Left panel: histogram data of OvinI $\lambda 18.969$ with its adjacencies in the observed wavelength space. The model and the continuum used are superimposed in red. Right panel: Velocity spectra with two vertical dashes lines at velocity zero and $1 / 2 w_{1}$. 


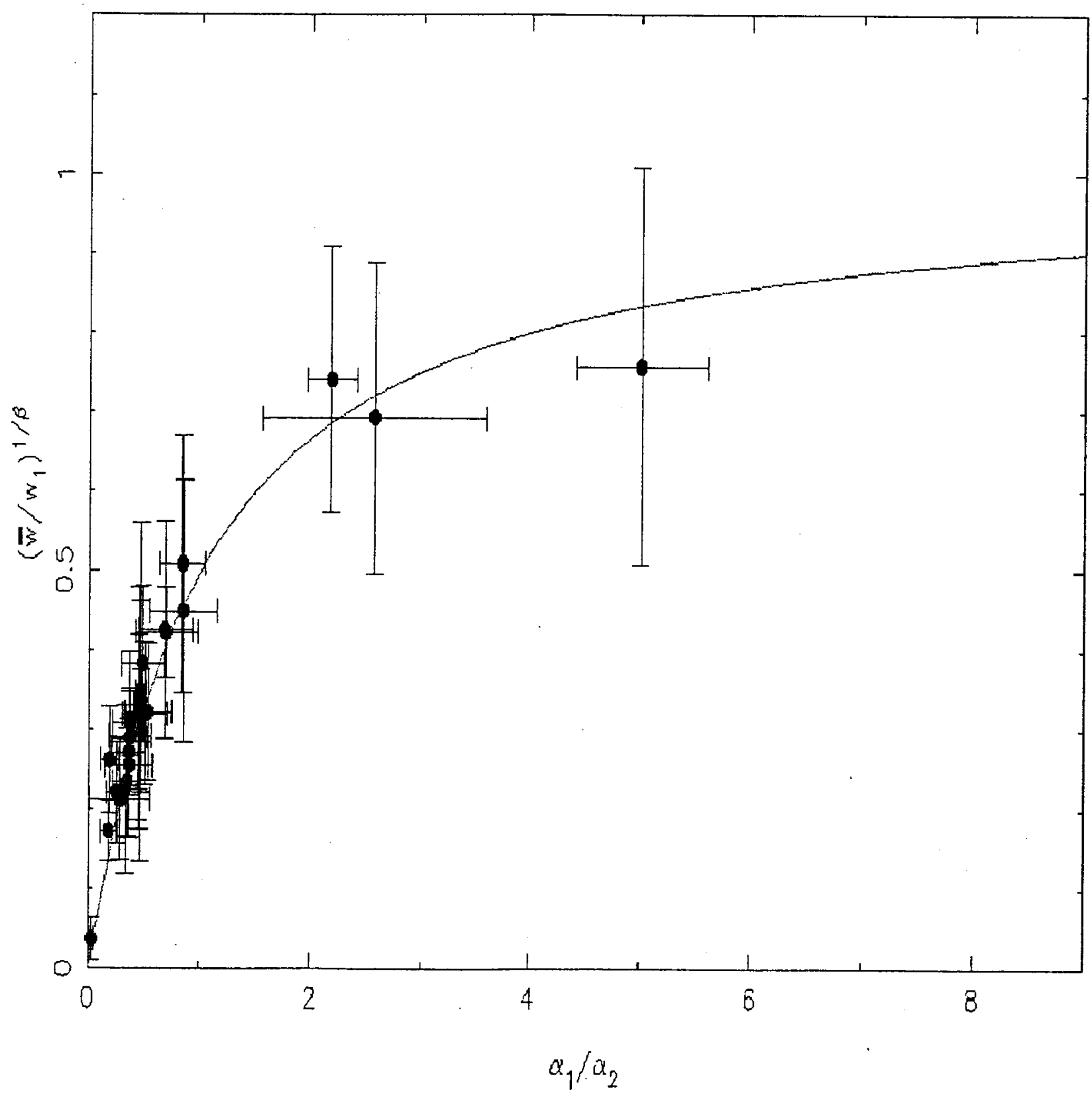

Fig. 6.- Distribution of the asymmetry for the resonance lines in the X-ray spectrum of NGC 3783 . 


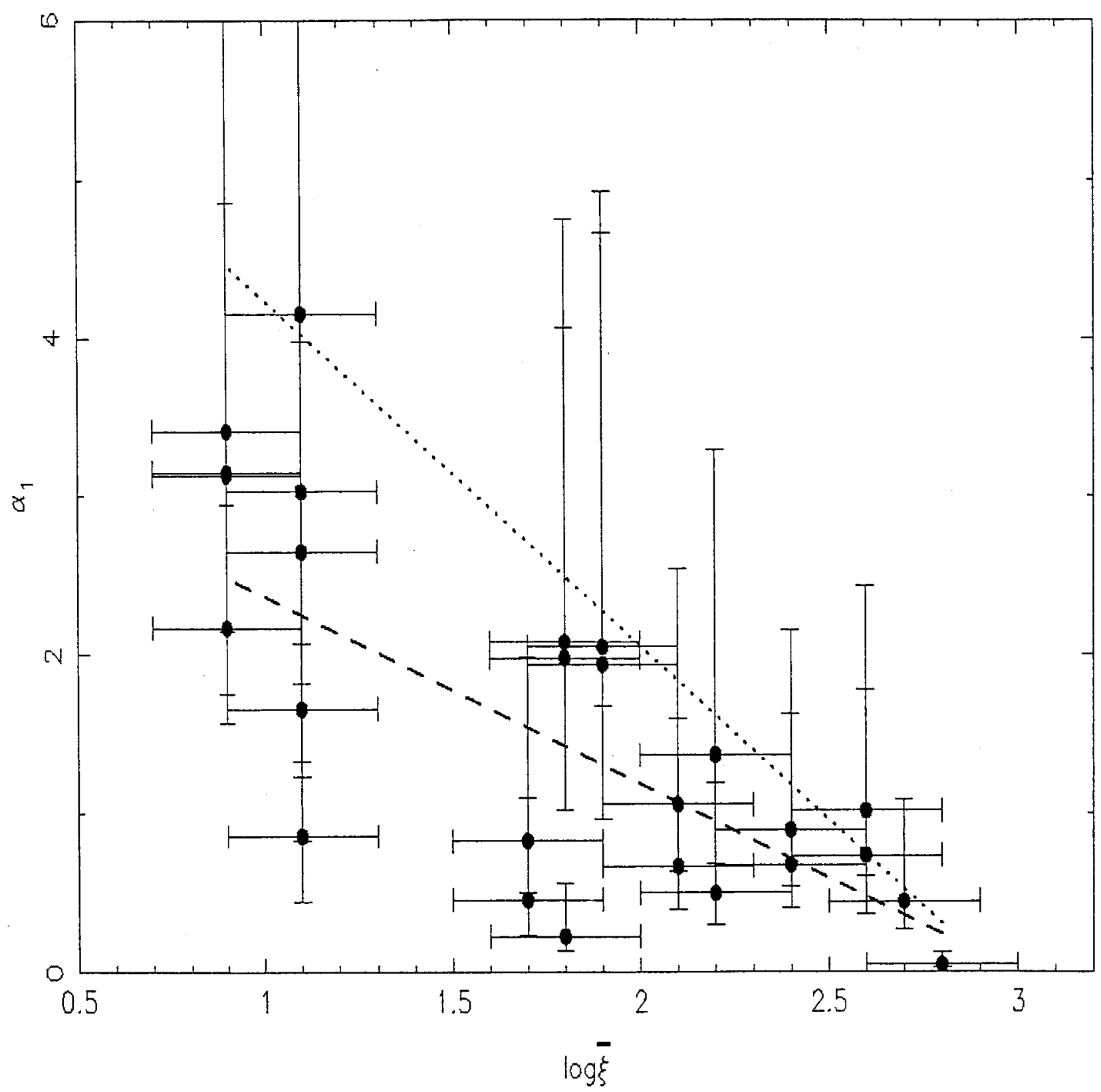

Fig. 7.- $\alpha 1$ vs. $\log (\bar{\xi})$. Error bars show values from table 3, long dashed lines show best fit linear regression, short dashed line is analytic model. 


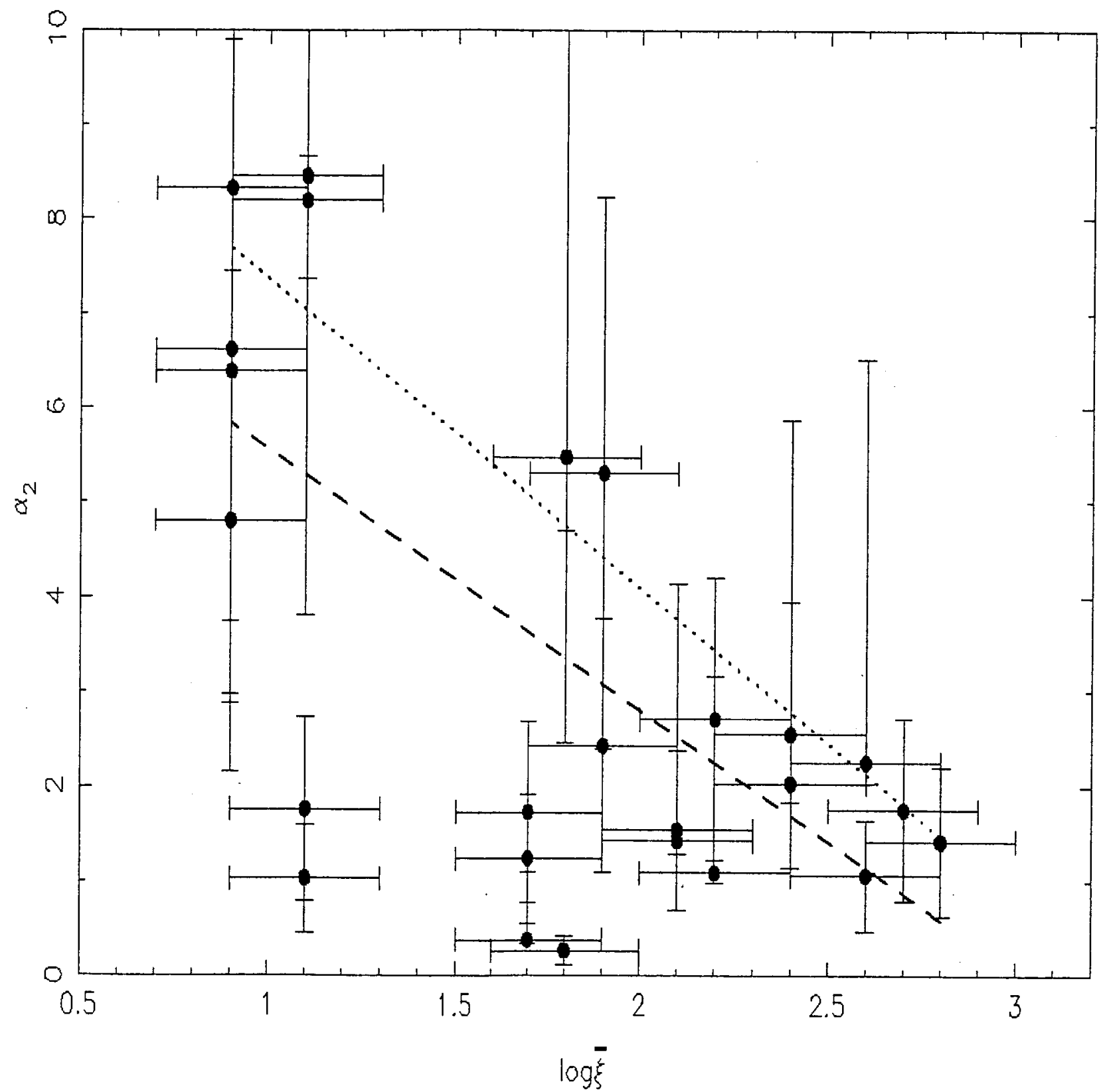

Fig. 8.- $\alpha_{2}$ vs. $\log (\bar{\xi})$. Error bars show values from table 3 , long dashed lines show best fit linear regression, short dashed line is analytic model. Note the differing vertical scale between from the previous figure. 


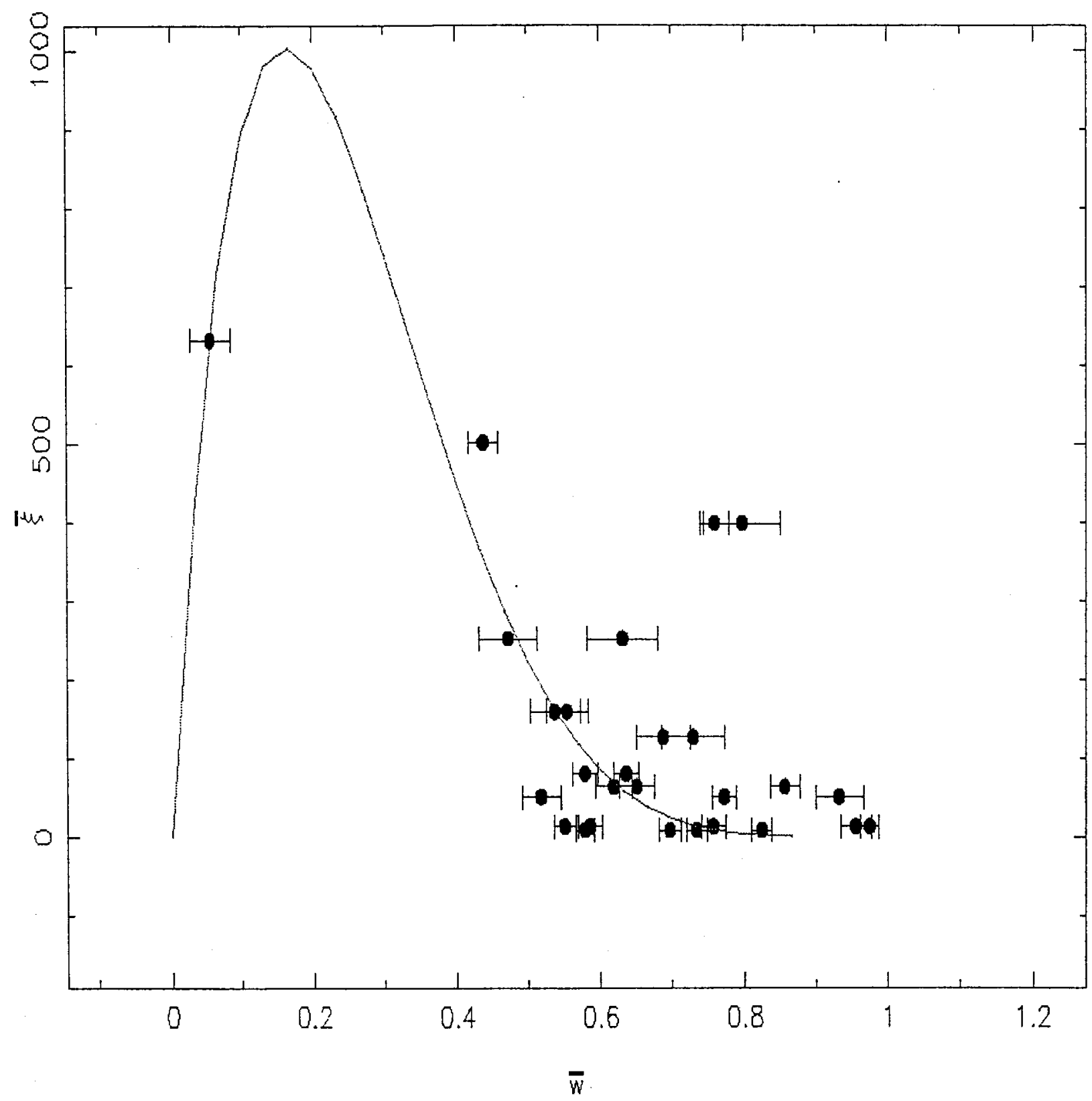

Fig. 9.- Ionization parameter where the absorption is maximum $\bar{\xi}$ vs the Doppler-shift velocity normalized to a terminal velocity of $1000 \mathrm{~km} / \mathrm{s} \bar{w}$. The predicted model in red. 